\title{
Gallstone Ileus in an Adult Male Patient: Computed Tomographic Findings and Case Report
}

Sule MB ${ }^{1 *}$, Joshua GK ${ }^{2}$, Dalha A ${ }^{2}$, Ribah MM ${ }^{2}$, Gidado $\mathrm{S}^{2}$, Yisa CB ${ }^{2}$, Aliyu AZ ${ }^{2}$.

${ }^{1}$ Radiology Department, Usmanu Danfodiyo University, Sokoto.

${ }^{2}$ Radiology Department, Usmanu Danfodiyo University Teaching Hospital, Sokoto.

*Corresponding Author: Sule Muhammad Baba; Department of Radiology, Usmanu Danfodiyo University, Sokoto.

Received date: April 19, 2021; Accepted date: May 22, 2021; Published date: May 31,2021

Citation: Sule MB, Joshua GK, Dalha A, Ribah MM, Gidado S, et al. (2021) Gallstone Ileus in an Adult Male Patient: Computed Tomographic Findings and A Case Report. J Thoracic Disease and Cardiothoracic Surgery, 2(2); DOI: 10.31579/2693-2156/021

Copyright: (C) 2021, Sule Muhammad Baba, This is an open access article distributed under the Creative Commons Attribution License, which permits unrestricted use, distribution, and reproduction in any medium, provided the original work is properly cited.

\section{Abstract}

Gallstone ileus is a mechanical intestinal obstruction due to gallstone impaction within the gastrointestinal tract, and responsible for less than $1 \%$ of cases of intestinal obstruction.

This is a case of a 50-year-old male who was referred for computed tomography of the abdomen on account of abdominal pain, abdominal swelling and discomfort, restlessness, vomiting, constipation, easy fatigability, excess sweating and restlessness.

The scout image of the computed tomography (CT) demonstrated linear streaks and foci of radiolucency beneath and around the $10^{\text {th }}$ and $11^{\text {th }}$ anterior ribs on the right most likely pneumobilia. Air distended bowel loops were also demonstrated. Serial axial slides demonstrated an impacted calculus measuring about $30 \mathrm{~mm}$ in widest transverse diameter in the right iliac region most probably the ileocecal valve region extending in to the cecum with associated dilated bowel loops showing multiple air-fluid levels raising a suspicion of mechanical bowel obstruction. The gall bladder measures about $50 \mathrm{~mm} \times 30 \mathrm{~mm}$ in cranio-caudal and mediolateral diameter, with turbid contents and thickened walls of about 4mm most likely from cholecystitis. A diagnosis of mechanical bowel obstruction following an impacted calculus at the ileocecal valve was entertained; Gallstone ileus in a 50-year-old man.

The patient had surgical treatment, this comprised of exploratory laparotomy with entero-lithotomy, fistula repair and cholecystectomy. The patient did well post-operatively and was discharged home subsequently and advised on follow-up visits.

We present a case of mechanical bowel obstruction following an impacted gall stone at the ileocecal valve in a 50-year-old male patient.

Keywords: mechanical bowel obstruction; computed tomography; ileus; pneumobilia; cholelithiasis; gallstone impaction; terminal ileum; ileocecal valve

\section{Introduction}

Gallstone ileus is a mechanical intestinal obstruction due to gallstone impaction within the gastrointestinal tract, and responsible for about $1-4 \%$ of cases of intestinal obstruction and predominantly occurs in females [13].

The most common site of gallstone impaction is the terminal ileum and the ileocecal valve, this follows a biliary enteric fistula following inflammation, the gallstones must measure about $2-2.5 \mathrm{~cm}$ in diameter to cause obstruction [2-4]

Gallstone ileus has shown a constant incidence of 30-35 cases/1000000, admissions over a forty-five-year period and develops in $0.3-0.5 \%$ of patients with cholelithiasis $[1,5,6]$

Gallstone ileus is predominantly a geriatric disease, and as many as 80$90 \%$ of patients have concomitant medical illnesses which include hypertension, congestive cardiac failure, diabetes, chronic pulmonary disease and anemia [1,7].

This condition has predilection for older female subjects with cholelithiasis over their male counterparts $[7,8]$.

Gallstone ileus has nonspecific symptoms and sign, most patient do have signs of acute cholecystitis preceding symptoms and signs of intestinal obstruction which may be partial or complete $[1,9,10]$. The main clinical presentation of gallstone ileus often depends on the site of impaction and most times include abdominal pain, distension, nausea, vomiting and constipation $^{2}$.

Plain abdominal radiographs and computed tomography are important in establishing the diagnosis of gall stone ileus, though CT scan remains the gold standard with sensitivity and specificity rates of $3 \%$ and $100 \%$ respectively $[2,3]$. 
In 1941, Rigler et al [10] described four radiographic signs of gallstone ileus, these are; partial or complete intestinal obstruction, pneumobilia or contrast material in the biliary tree, an aberrant gallstone and change of position of the gallstone on serial films.

The presence of two of the three first radiographic signs has been considered pathognomonic and found in about $20-50 \%$ of cases $[1,11$, 12].

The study is aimed at demonstrating the computed tomographic features of gallstone ileus with mechanical obstruction.

\section{Case Report}

This is a case of a 50-year-old male civil servant who was referred from a health facility in the neighboring settlement for an abdominal computed tomographic scan on account of abdominal pain, abdominal swelling and discomfort, restlessness, vomiting, constipation, easy fatigability, excess sweating and restlessness. These were preceded by frequent history of right upper quadrant colicky abdominal pain and discomfort with occasional vomiting especially after fatty meals and milk containing feeds raising a suspicion of cholecystitis.

The patient appeared in painful distress, with tenderness over the right hypochondrial region and a positive Murphy's sign, not pale, anicteric, acyanosed, mildly dehydrated, no finger clubbing and no pedal swelling.

The blood pressure was about $110 / 65 \mathrm{mmHg}$, pulse rate was about 72 beats per minute, the respiratory rate was 12 cycles per minute. The blood electrolyte, blood urea and blood creatine levels were also normal; with values of about 12 milligram per deciliter and 1.1 milligram per deciliter of blood for urea and creatine respectively. The packed cell volume was about $38 \%$, the white blood cells were elevated and about 13500 white blood cells per microliter in favor of the lymphocytes having a count of about 6500 lymphocytes in 1 microliter of blood. The erythrocyte sedimentation rate was also normal and about 10 millimeters per hour. The liver function test was also normal for the patient's age.

The scout image of the computed tomography (CT) demonstrated linear streaks and foci of radiolucency beneath and around the $10^{\text {th }}$ and $11^{\text {th }}$ anterior ribs on the right most likely pneumobilia. Oval calcific density opacities with surrounding lucency were also demonstrated in the region of the right iliac fossa most likely calculi within the bowel loops. Air distended bowel loops were also demonstrated (Figure 1). Serial axial slides demonstrated an impacted oval hyper-density measuring about $30 \mathrm{~mm}$ in widest transverse diameter most likely a calculus in the right iliac region most probably the ileocecal valve region extending in to the cecum (Figures 2\&3) with associated dilated bowel loops showing multiple air-fluid and air-contrast levels raising a suspicion of mechanical bowel obstruction (Figures $4 \& 5$ ). The other calcific density areas were noted within the bowel loops and appear not impacted. The gall bladder measures about $50 \mathrm{~mm} \times 30 \mathrm{~mm}$ in cranio-caudal and mediolateral diameter, with turbid contents and thickened walls of about $4 \mathrm{~mm}$ most likely from cholecystitis (Figure 6). No gall bladder calculus was demonstrated. Hepatomegaly with a liver span of about $159 \mathrm{~mm}$ was also demonstrated (Figure 5). The pancreas was also demonstrated, it showed normal size and outline with no calculus within its ducts and no feature to suggest pancreatitis noted. A diagnosis of mechanical bowel obstruction following an impacted calculus at the ileocecal valve was entertained; Gallstone ileus in a 50-year-old man.

The patient had surgical treatment, this comprised of exploratory laparotomy with entero-lithotomy, fistula repair and cholecystectomy. The patient did well post-operatively and was discharged home subsequently and advised on follow-up visits.

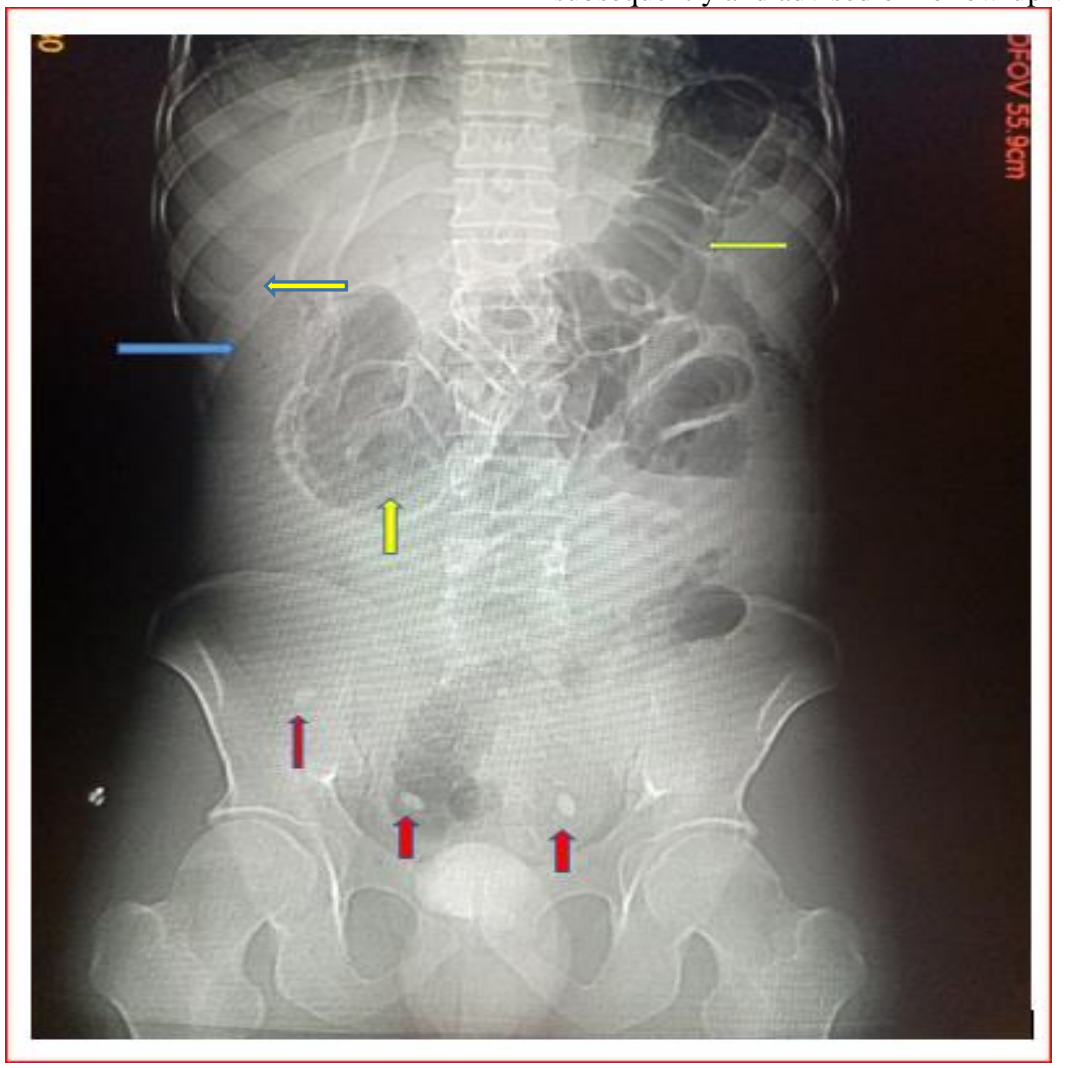

Figure 1: Scout image of a CT scan demonstrating streak and foci of lucency beneath the 10th rib and within the 11th intercoastal space on most likely pneumobilia. Three oval radio-opacities of calcific density are demonstrated distally in the pelvic region with surrounding lucency most likely in the bowel lumen. The demonstrated bowel loops show luminal prominence. The demonstrated bones show degenerative changes. 


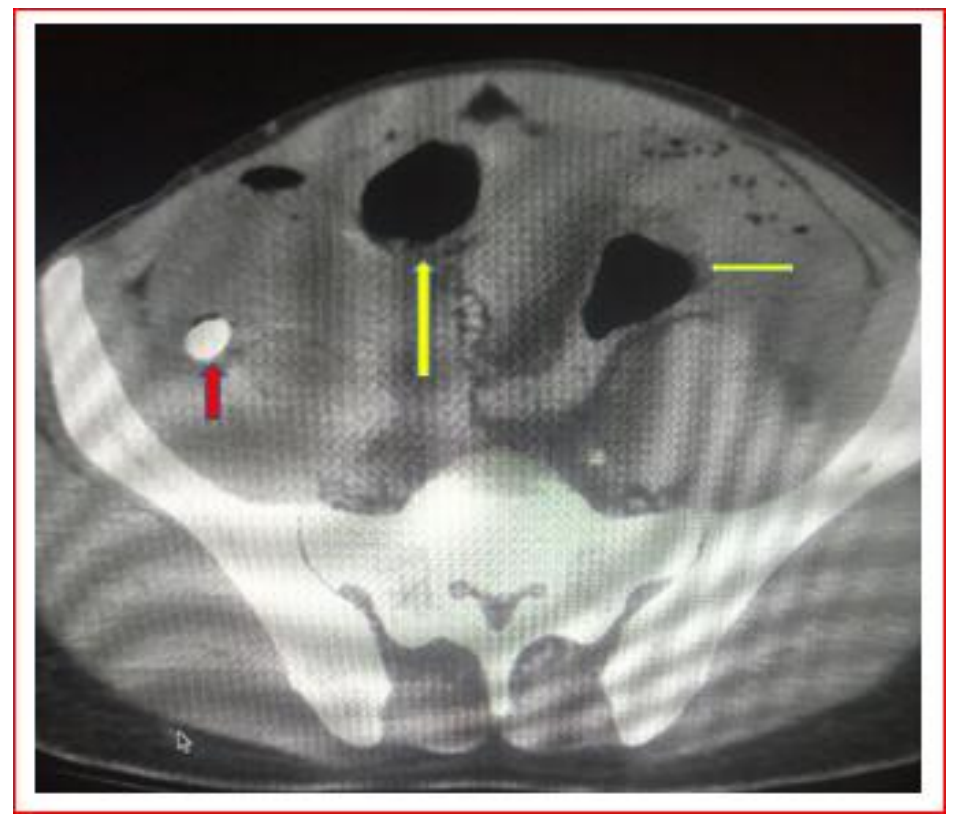

Figure 2: An axial serial non-contrast enhanced image of the lower abdomen/pelvis demonstrating an oval hyper-density measuring about 30mm in widest transverse diameter in the bowel loop located at the right iliac fossa; most likely the gallstone (red up arrow). The demonstrated bowel loops are distended (yellow up and left arrows).

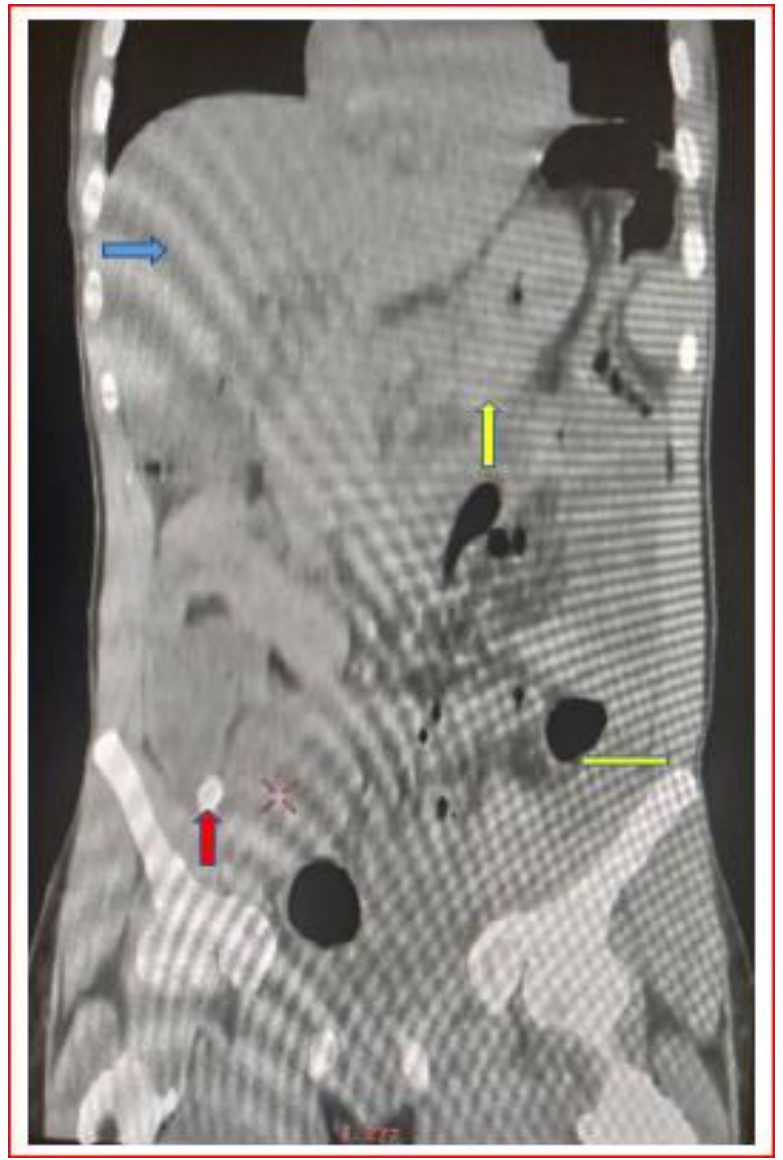

Figure 3: A constructed coronal serial non-contrast enhanced image of the abdomen and pelvis demonstrating an oval hyper-density measuring about $30 \mathrm{~mm}$ in widest transverse diameter in the bowel loop located at the right iliac fossa; most likely the gallstone (red up arrow): note the transition of the diameter of the bowel loops; smaller medially and larger laterally beyond the calculus most likely the ileocecal region. The demonstrated bowel loops are distended (yellow up and left arrows) with an enlarged liver; span is $159 \mathrm{~mm}$ (blue right arro 


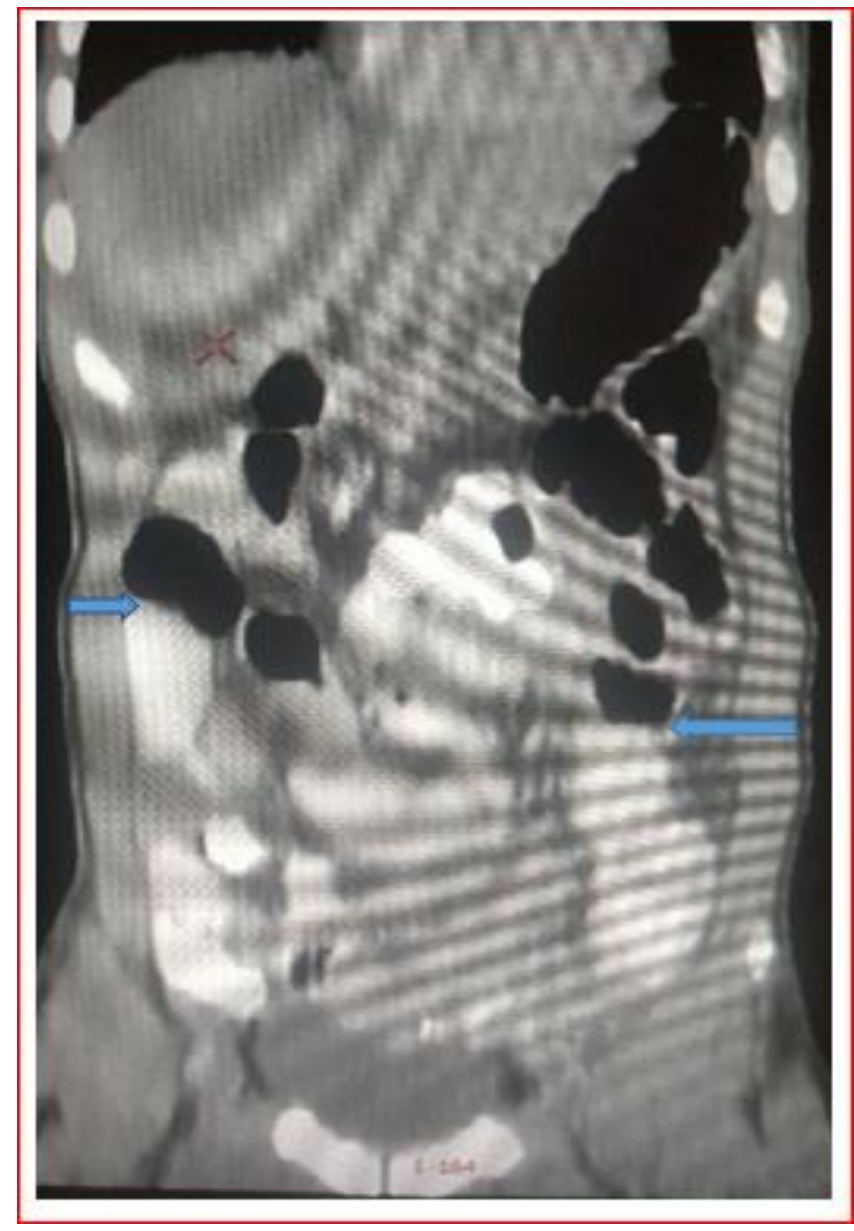

Figure 4: A post oral reconstructed coronal image of the CT scan demonstrating dilated bowel loops with air-contrast level (right and left blue arrows) in both hemi-abdomen in keeping with mechanical dilatation; obstruction. The calculus is however masked by the oral contrast.

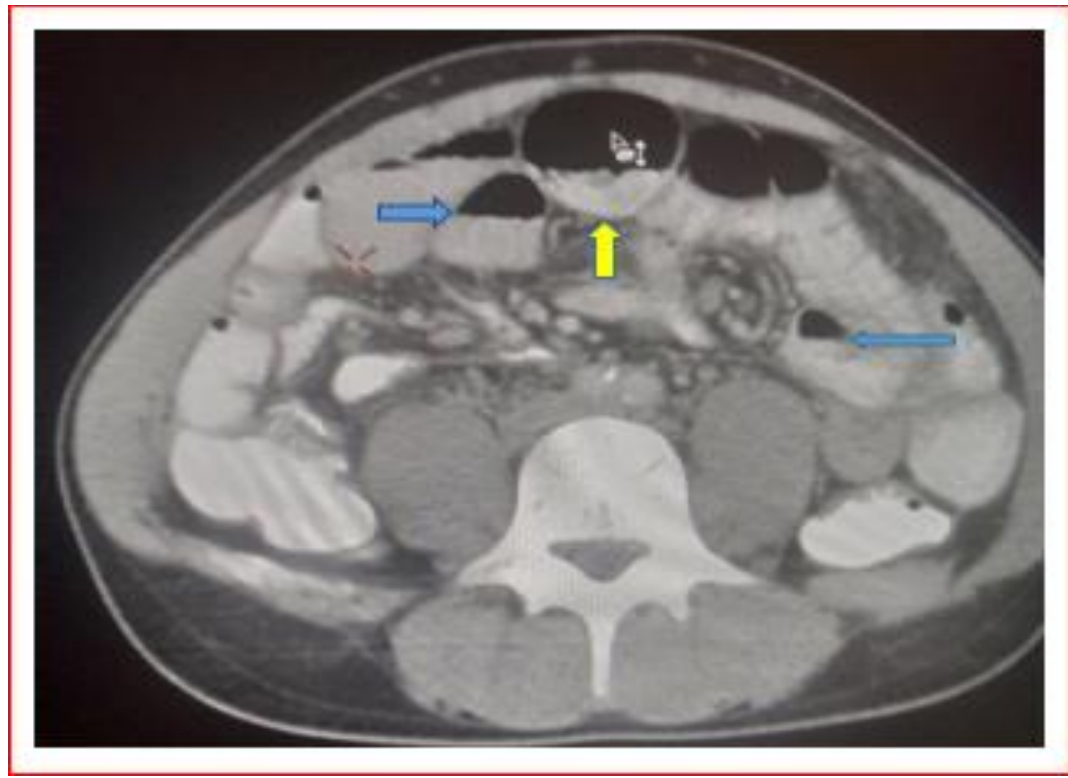

Figure 5: A post oral contrast axial image of the CT scan demonstrating dilated bowel loops with air-contrast level in the central (yellow up arrow), and both hemi-abdomen (right and left blue arrows) in keeping with mechanical dilatation; obstruction. 


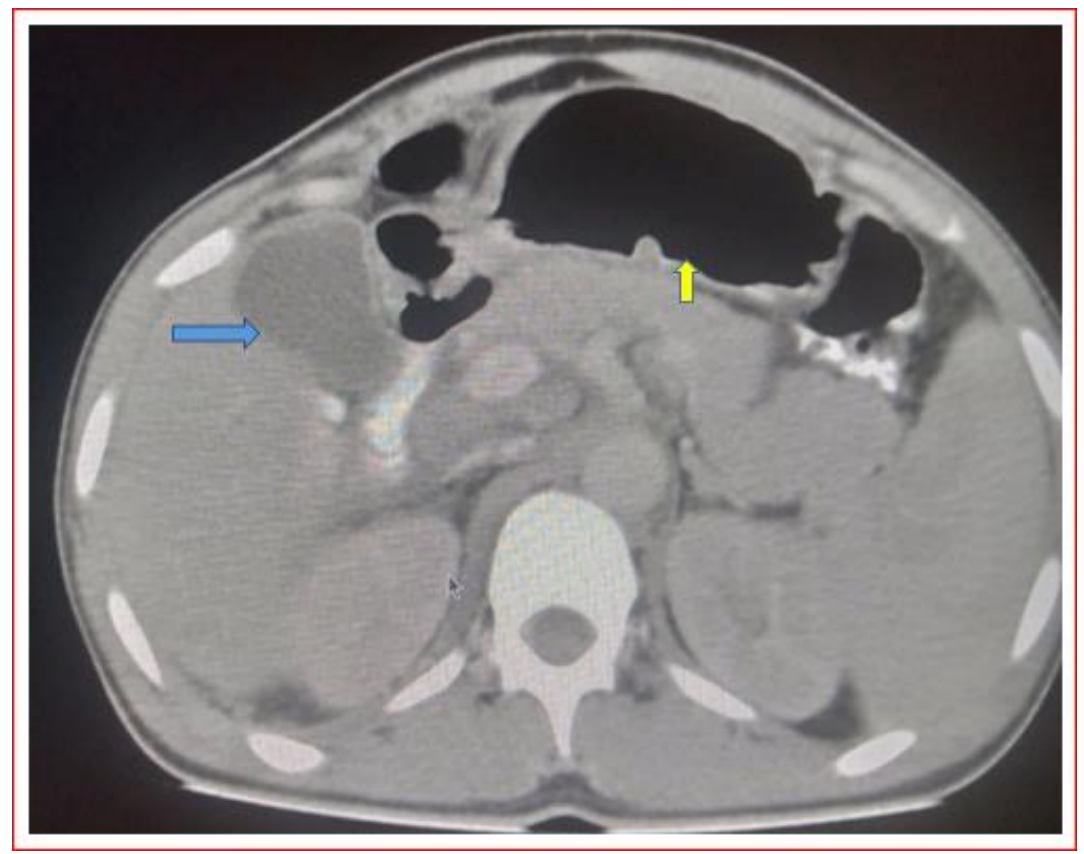

Figure 6: An axial post-oral contrast series; early image demonstrates a thick-walled $(4 \mathrm{~mm})$ gall bladder measuring about 50mm x $30 \mathrm{~mm}$ in craniocaudal and mediolateral dimension with slightly turbid content most likely from cholecystitis. The bowel loops are gas distended.

\section{Discussion}

Gallstone ileus is a disease of the elderly and more frequently seen among the female subjects [1-3], the index case is a 50-year-old conforming to that reported in the literature but happens to be a male patient contrary to the documented prevalence in the female gender.

Gallstone ileus is a rare disease and accounts for $1-4 \%$ of mechanical obstruction, prevalent in the elderly with associated high mortality rate, and difficult diagnosis, though early diagnosis however reduces the mortality [13]. The index case was however diagnosed early following imaging, with prompt institution of managing thereby conforming to this literature.

The most common site of gallstone impaction is the terminal ileum and the ileocecal valve, this follows a biliary enteric fistula following inflammation, the gallstones must measure about $2-2.5 \mathrm{~cm}$ in diameter to cause obstruction [2-4]. The index case had an enteric calculus impacted at the ileocecal region/valve and measures about $30 \mathrm{~mm}$ in widest diameter, thereby conforming to these literatures.

Most literatures document the preceding features of symptoms of cholecystitis in individuals with gallstone ileus $[1,5,6]$, the index case also had features highly suspicious of cholecystitis conforming to that documented in the literature.

Patients presenting with features of gallstone ileus do occasionally have associated ailments like hypertension, diabetes mellitus to mention a few $[1,7]$, the index case had no comorbidity invariance to these documented literatures.

The classical presenting features of abdominal pain and discomfort, abdominal distension and associated features of intestinal obstruction are those documented in the literature $[1,2,9,10]$, the patient also presented with these features conforming to these literatures.

Plain radiograph and $\mathrm{CT}$ go a long way in establishing the diagnosis of gallstone ileus, though CT is the gold standard modality in establishing this diagnosis as mentioned by most researchers [2,3], the index case was also diagnosed following clinical presentation and CT scan of the abdomen conforming further to that documented in these literatures.

The classical radiographic features and finding of distended bowel loops, gallstone within the bowel lumen, pneumobilia and free intraperitoneal air with bowel loops having air-fluid levels are those documented in the literatures $[2,3,10]$, these plain radiographic findings were also demonstrated in the index case conforming to that documented in the literature.

Rigler et al [10] described four radiographic signs of gallstone ileus, these are; partial or complete intestinal obstruction, pneumobilia or contrast material in the biliary tree, an aberrant gallstone and change of position of the gallstone on serial films. The presence of two of the three first radiographic signs has been considered pathognomonic and found in about $20-50 \%$ of cases $[1,11,12]$. The case under review had features of intestinal obstruction, pneumobilia, and presence of an impacted calculus at the right iliac fossa; ileocecal valve region, thereby conforming to these literatures.

Patients with gallstone ileus do have surgical intervention as a treatment modality as documented in the literature [2], the index case also had surgical operation; exploratory laparotomy with cholecystectomy as treatment option further conforming to most literatures.

\section{Conclusion}

Computed tomographic scan of the abdomen should be carried out on patients with high suspicious of gallstone ileus as it regarded the gold standard in establishing such diagnosis for prompt institution of management with subsequent reduction in morbidity and mortality associated with the condition.

\section{References}

1. Carlos MNG, Maria EMC, Jorge LC.(2016) Gallstone ileus, clinical presentation, diagnostic and treatment approach. World $J$ Gastrointest Surg.; 8:65-76.

2. Teixeira C, Alves AL, Cremers I.(2018) Colonic Gallstone ileus: A rare case. GE Port j Gastroenterol.. DOI:10.1159/000485804. 
3. Clavian PA, Richon J, Burgan S, Rohne A.(1990) Gallstone ileus. Br J Surg. 77:737-742.

4. Reisner R, Cohen J(1994) Gallstone ileus: a review of 1,001 reported cases. Am Surg.; 63:441-446.

5. Kurtz RJ, Heimann TM, Beck AR, Kurtz AB.(1985) Patterns of treatment of gallstone ileus over a 45-year period. Am J Gastroenterol. 80:95-98.

6. Halabi WJ, Kang CY, Ketana N, Lafaro KJ, Nguyen VQ, Stamos MJ, et al.(2014) Surgery for gallstone ileus: a nationwide comparison of trends and outcomes. Ann Surg.; 259:329-335.

7. Ayatunde AA, Agrawal A. (2007)Gallstone ileus: diagnosis and management. World J Surg. 31:1292.

8. Kasahara Y, Umemura H, Shiraha S, Kuyama T, Sakata K, Kubota H.(1980)Gallstone ileus. Review of 112 patients in the Japanese literature. Am J Surg. 140:437-440.

9. Zaliekas J, Munson JL.(2008) Complications of gallstones: the Mirizzi syndrome, gallstone ileus, gallstone pancreatitis, complications of lost gallstones. Surg Clin North Am. 88:13451368.

10. Rigler LG, Borman CN, Noble JF.(1941) Gallstone obstruction: pathogenesis and roentgen manifestations. JAMA.; 117:17531759.

11. Abou-Saif A, Al-Kawas FH.(2002) Complications of gallstone disease: Mirizzi syndrome, cholecystocholedochal fistula, and gallstone ileus. Am J Gastroenterol. 97:249-254

12. Luu MB, Deziel DJ. (2014) Unsual complications of gallstones. Surg Clin North Am.;94:377-394.

13. Jen-Wei Chou, Chang-Hu Hsu, Kuan-Fu Liao, Hsueh-Chou Lai, Ken-Sheng Cheng, Cheng-Yuan Peng, Mei-Due Yang, and YungFang Chen. (2007) Gallstone ileus: Report of two cases and review of the literature. World J Gastroenterol. Feb 28; 13(8): 1295-1298.
This work is licensed under Creative Commons Attribution 4.0 License

\section{To Submit Your Article Click Here: Submit Manuscript}

DOI: $10.31579 / 2693-2156 / 021$
Ready to submit your research? Choose Auctores and benefit from:

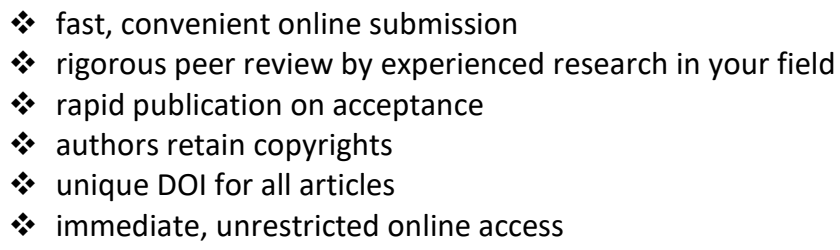

At Auctores, research is always in progress.

Learn more www.auctoresonline.org/journals/journal-of-thoracicdisease-and-cardiothoracic-surgery- 